\title{
Emerging infections and the integrative environment-health sciences: the road ahead
}

Matthew C. Fisher(i) ${ }^{1 凶}$ and Kris A. Murray $\mathbb{1}^{1,2}$

The integrative environment-health sciences including One Health, Conservation Medicine, EcoHealth and Planetary Health embody the transdisciplinary synthesis needed to understand the multitude of factors that underpin emerging infections and their management. Future successes in confronting and resolving the complex causal basis of disease emergence to generate robust, systems-oriented risk reduction strategies that preserve both human health as well as promoting sustainable futures represent the 'Moon Shot' for the integrative environment-health sciences.

The 'Globalization Big Bang' has, on one hand, advanced public health through the successes of industrialization, mobility and technology while, on the other, has created new global health challenges associated with rapid rates of sociological, biological and environmental change. The integrative environment-health sciences (IEHS), including One Health, Conservation Medicine, EcoHealth and Planetary Health, view human health as interdependent with that of other living species and our shared environment. From their origins in 'ecological public health' and 'one medicine' in the mid-twentieth century, the IEHS are now represented within many of the world's highest-level international policies, including the United Nations Sustainable Development Goals, post-2020 Biodiversity Framework and the Paris Agreement. The current incarnation of this approach is conceptualized as a systems-facing transdisciplinary framework aimed at maximizing global health by protecting the functioning systems of the planet itself.

Biodiversity is a pillar of resilient ecosystems via the preservation of a myriad functional interactions and genetic reserves reflecting billions of years of evolutionary history. However, biological diversity at the microorganism-environment-human interface presents a perennial risk as sylvatic reservoirs of potentially novel human pathogens. The majority of human endemic and emerging infections are caused by pathogens shared with other animals. This interconnectivity of humankind to the biodiversity with which we share the planet becomes cruelly apparent when outbreaks of zoonotic infections occur, some of which go on to cause epidemics and, rarely but very impactfully, pandemics. This has never been plainer than in 2020 when viewed across a world locked down by SARS-CoV-2. In the same vein, the IEHS illuminate the eco-evolutionary impacts of on-target and off-target chemical drug exposure in agriculture and animal husbandry. The intensive production of the foods and fibres that we are dependent upon is linked to the declining efficacy of our clinical armamentarium via spillover across the microorganism-environment-human interface.

We here laud the fundamental goals of the IEHS as a conceptual approach within which to foster interdisciplinary collaboration and frame research and policy agendas. However, we (and others) question the progress that has been made in crafting these disciplines into operational protocols to prevent emerging infections. Simply put, business-as-usual as well as crisis health care, food security and nature conservation proceeds primarily as siloed decision-making in response to proximal events (outbreaks, famines and species loss) without routinely taking into consideration the distal causes (socio-ecological relationships) of these events. This is not surprising - the challenge of understanding complex socio-ecological systems in a manner that allows us to understand, let alone predict, the risk of disease emergence without overwhelming uncertainty, is formidable. The downstream translation of a scattered evidence base comprising variable quality data into widely adoptable, cost-effective and socially and politically acceptable policies, is harder still.

Yet this is precisely the task these disciplines have taken aim at, with stakeholders across these realms delivering strong advocacy or policy suggestions that promise to reduce future disease emergence risks, primarily via improving environmental stewardship. In the wake of COVID-19, for instance, there has been no shortage of fingers pointed, policies drafted, and position papers prepared that aim squarely at banning the wildlife trade, bushmeat hunting or deforestation. However, as seen for a panoply of pathogens before, 
actionable and sustainable policies to reduce the risks of future disease emergence remain too broadly cast and seem likely to fade into the background as SARS-CoV-2 is brought under control.

The key issues facing the IEHS as a stewarding force and source of genuinely novel inspiration that goes beyond existing paradigms are those of how to translate scientific advances into transnational narratives forged, promises sustained, and both ecosystem and health-care systems strengthened. Spillover, for example, is not an uncommon process, and preventing it entirely would require complete and unnecessary decoupling of humans from other animals and nature more broadly. This is far too wide a target for emergence- or pandemic-prevention that could itself have many unintended consequences. We believe that a critical dislocation between the theory and practice of the IEHS as they relate to emerging infections is a widespread lack of specificity in linking environmental 'actions' to clinically relevant disease emergence risks. This results in an overreliance on (and in some cases the over-sale of) generic narratives on which governments and other stakeholders are required to primarily invoke the 'precautionary principal' if they wish to try to reduce the apparent risks. This may be appropriate in some cases, but in our view more often presents a barrier for inaction, encourages policies with limited potential for real-world implementation or prospects for efficacy evaluation, or increases the chance of collateral damage, all of which ultimately undermine their sustainability. Some examples include:

1. Although the emergence of Planetary Health as a 'new' discipline in 2014 (REF.) signifies a broadening of One Health and an entry point for many medical professionals to identify with and engage in the IEHS, there remains a chasm between ambition and actionable evidence across these disciplines that cannot be filled by narrative alone. For instance, only $8 \%$ of planetary health publications are currently considered 'original research' ${ }^{2}$, whereas the vast majority ( $90 \%)$ are opinion pieces, commentaries, calls to action, syntheses, reviews and framework suggestions. The energy, participation, ability to synthesize, and increased medically trained stakeholder base are hugely valuable, yet clearly there is a need for more primary research and innovation to make genuine progress.

2. Despite integrative recognition of the disease risks incurred by land-use change and biodiversity loss made at the turn of the century ${ }^{3}$, two decades later such findings remain contentious ${ }^{4}$ and predominantly notional from a health protection or co-benefits perspective; it is still not possible to routinely incorporate the risks from infectious diseases into land-use change or agricultural policies alongside the protection of biodiversity, in any operational sense. This is despite the parallel advancement of ecosystem services and natural capital accounting methods that seek and, in many cases, succeed in specifying both monetary and non-monetary or indirect benefits of nature known to influence policy making.
3. Although some proponents advocate for simple blanket policies, such as wildlife trade or deforestation bans, to reduce disease emergence risks en masse, it is insufficient for these to be poorly characterized with respect to the emergence prevention value that they represent, particularly where significant costs (for example, to traditional livelihoods, driving trade underground and risking food insecurity) can be anticipated. Innovation in developing systemsbased approaches aimed at better understanding and quantifying the costs versus the benefits of policy interventions that seek to curb 'risky' human endeavours are required. For instance, small-hold mixed-species farms coupled with relatively inefficient domestic market chains may increase zoonotic disease emergence risks due to enhancing the chance of cross-species pathogen transmission. However, the alternatives may not fare much better - for instance larger scale, more industrial food production and trade may be more biosecure but still fail to eliminate disease emergence risks and can have larger negative nutritional, ecosystem or climate impacts.

4. Despite developments in resolving the mechanisms of emergence at the microorganism-environmenthuman interface, 'stopping disease emergence at source' in what is increasingly being touted the 'Age of Pandemics' remains an attractive but oversimplified deliverable perennially disconnected from the evidence base. Whereas several proponents regard the emergence of SARS-CoV-2 as a largely predictable next chapter, current assertions regarding the increasing rate of novel disease emergence, particularly from viral zoonoses, do not square with the relatively linear or even declining rates of zoonotic viral discovery documented in humans ${ }^{5}$. Furthermore, spatial models of wildlife-origin zoonotic emergence suggest predictive skill that may be only marginally better than random ${ }^{6}$, while methodologically remaining deeply confounded by our inability to disentangle improving skill in detecting emerging infections from increasing observations of their occurrence. Similarly, efforts to establish the highest risk zoonotic reservoir taxa continue to be challenged ${ }^{7}$, while estimates of the unknown microbial diversity that could be the source of future zoonoses differ by several orders of magnitude ${ }^{8}$. Such limitations have large, uncomfortable implications for proposed pre-emptive surveillance programmes (for example, REF. ${ }^{9}$ ) that rely on these measures of 'the where, when and what' of disease emergence to be transparently costed, adequately targeted and potential efficacy evaluated. Cycles of scientific advance, revision and refinement are necessary, and these are all important scientific endeavours to which we have ourselves contributed, but we can ill afford to direct scarce disease emergence-prevention resources where we risk failing to deliver appreciable gains in risk reduction or health protection.

5. The intersection between climate science, policy and governance has arguably become the benchmark of how to mobilize and justify global actions that seek to limit environmental harms and their societal impacts. However, and as the climate change and COVID-19 
conspiracy narratives have all too clearly shown, we still lack the behavioural science and the political will needed to implement scientific evidence or consensus into actionable policy and societal acceptance within the relevant timescales.

Although operationalizing IEHS approaches in a manner that achieves its full potential remains a daunting task, there are welcome signs that concerted global action to tackle global health threats is possible, some of which have been reflected in the current COVID-19 pandemic. Despite association with enormous societal and economic cost, 'lockdowns' have directly saved millions of lives by avoiding the high attack rates that are seen in unmitigated epidemics. Lockdowns are only viable in the short term in order to allow a window of opportunity within which exit strategies can be developed and yet illustrate the potential for human societies to coordinate on a truly grand scale, backed by science, to confront emerging health crises. First community use of the BNT162b2 mRNA vaccine in a non-trial individual occurred only 342 days after the Wuhan Municipal Health Commission reported the initial SARS-CoV-2 cluster. Following unprecedented speed in viral sequencing to identify SARS-CoV-2 as a newly emerged pathogen and chronicling its global spread, that vastly accelerated drug-development and distribution pipelines are achievable if social, political and financial support is available, charts the way forward for investing in the rapidly scalable pipelines that will continue to be our frontline defence against future transboundary infections.

Some of these emergency measures bring in to sharp focus our potential to cooperatively tackle arguably much larger health crises in the making, such as the emissions reductions required to minimize the human health harms of global climate change, the shifts in land use required to limit global biodiversity and ecosystem services loss on which we still ultimately depend, and perhaps even the nuances of emerging infections and other future pandemics more generally. Clearly, the timescales over which such crises unfold rather than their projected magnitude in terms of health threat is one critical determinant of political and societal uptake, highlighting that economic discounting with time continues to blind in the face of slow moving and relatively complex catastrophes. Here, however, and despite some of the shortcomings touched upon above, the IEHS may hold the keys to unlocking this same global potential for the complex, interdisciplinary health threats that will come to define the Anthropocene.

Firstly, COVID-19 has won the argument that boosted research pipelines linked to Big Pharma scalability and equitable trans-national delivery are the route to a timely exit from pandemics. That our main armamentarium against COVID-19 (epidemiological analysis, drug/vaccine development, clinical trials and diagnostics) were spearheaded by biotech start-ups or universities underscores the dependency of operational IEHS upon a flourishing scientific infrastructure. Synchronously, big data and deep-learning computational approaches are rapidly scaling humans' ability to understand the scalar dimensions of our biotic and abiotic world. As our understanding improves through models, then science's ability to convey risk and uncertainty to decision-makers will increase. However (and secondly), for evidence-based decision-making to occur, then the outputs of science need to be heard. Philosophically, science jostles for intellectual space with many belief systems, and without carefully attending to the psychological, economic and political context within which science is delivered, informed decisions will not occur. Perennially, cognitive 'optimism bias' leads to an underappreciation of risk and uncertainty in governance, and the greater the extent that IEHS interlocks with policy, the greater its ability to inform decisions will be. Thirdly, operational IEHS requires coordinated transnational action, which requires scientific advances to not only be recognized, but to be implemented. Here, and despite the bumpy ride experienced across 2020, the World Health Organization and allied international policy instruments have functioned as they should. For lesser-studied but highly destructive wildlife diseases such as amphibian chytridiomycosis, the timeline of discovery through to actioned response has declined from decades to years ${ }^{10}$ leading to increased international biosecurity that has so far avoided further catastrophic declines of biodiversity. Finally, centuries of scientific research, technological advance and human-rights improvements have led to an unprecedented appreciation of the singularity that comprises the Earth biosphere. Looking back, 2020 may well mark a threshold where the IEHS gains traction as an operational protocol-based paradigm. Victories are certainly possible, and the advancement of germ theory has led to many wars being won against infection. However, what lies ahead is a dance rather than a war and the success of IEHS will be determined by fleetness of foot rather than by massed artillery, taking the form of unprecedented enquiry into the mechanics and integrated management of increasingly unstable socio-ecological systems.

1. Horton, R. et al. From public to planetary health: a manifesto. Lancet 383, 847 (2014).

2. Rossa-Roccor, V. et al. Scoping review and bibliometric analysis of the term "planetary health" in the peer-reviewed literature. Front. Public Health 8, 343 (2020).

3. Patz, J. A. et al. Unhealthy landscapes: policy recommendations on land use change and infectious disease emergence. Environ. Health Perspect. 112, 1092-1098 (2004).

4. Rohr, J. R. et al. Towards common ground in the biodiversity-disease debate. Nat. Ecol. Evol. 4, 24-33 (2020).

5. Rosenberg, R., Johansson, M. A., Powers, A. M. \& Miller, B. R. Search strategy has influenced the discovery rate of human viruses. Proc. Natl Acad. Sci. USA 110, 13961-13964 (2013).

6. Allen, T. et al. Global hotspots and correlates of emerging zoonotic diseases. Nat. Commun. 8, 1124 (2017).

7. Mollentze, N. \& Streicker, D. G. Viral zoonotic risk is homogenous among taxonomic orders of mammalian and avian reservoir hosts. Proc. Natl Acad. Sci. USA 117, 9423-9430 (2020).

8. Carlson, C. J., Zipfel, C. M., Garnier, R. \& Bansal, S. Global estimates of mammalian viral diversity accounting for host sharing. Nat. Ecol. Evol. 3, 1070-1075 (2019).

9. Carroll, D. et al. The Global Virome Project. Science 359, 872-874 (2018).

10. Fisher, M. C. \& Garner, T. W. J. Chytrid fungi and global amphibian declines. Nat. Rev. Microbiol. 18, 332-343 (2020).

\section{Acknowledgements}

M.C.F. and K.A.M. were funded by the UK Medical Research Council and UK Natural Environmental Research Council. M.C.F. is a CIFAR Fellow.

\section{Competing interests}

The authors declare no competing interests. 